2. M. Deuring, Algebren, Ergebnisse der Mathematik und ihrer Grenzgebiete, vol. 4, Berlin, Springer, 1935.

3. G. Hochschild, On the structure of algebras with nonzero radical, Bull. Amer. Math. Soc. vol. 53 (1947) pp. 369-377.

4. - Double vector spaces over division rings, Amer. J. Math. vol. 51 (1949) pp. 443-460.

5. N. Jacobson, A note on division rings, Amer. J. Math. vol. 49 (1947) pp. 27-36.

UNIVERSITY OF ILLINOIS

\title{
ON THE RADICAL OF A LIE ALGEBRA
}

\section{HARISH-CHANDRA}

Let $\mathfrak{R}$ be a Lie algebra over a field $K$ of characteristic zero. For any $X \in \mathbb{R}$ we denote, as usual, the linear mapping $Y \rightarrow[X, Y]$ of $\mathbb{R}$ into itself by ad $X$. Let $\Gamma$ be the radical of $\mathfrak{R}$. Consider the set $\mathfrak{R}$ consisting of all $N \in \Gamma$ such that ad $N$ is nilpotent. It was shown in a recent paper ${ }^{1}$ that $\mathfrak{N}$ is the unique maximal nilpotent ideal ${ }^{2}$ of $\mathfrak{R}$. Further if $D$ is a derivation of $\Gamma$ then $D \Gamma \subset \Re$.

For any $X, Y, Z \in \mathbb{R}$ put $B(X, Y)=s p(\operatorname{ad} X$ ad $Y)$ and $T(X, Y, Z)$ $=s p(\operatorname{ad}[X, Y] \operatorname{ad} Z)$. Then $B(X, Y)$ is a symmetric bilinear form on $\&$ while $T(X, Y, Z)$ is a skewsymmetric trilinear form. It is easily verified that they are both invariant under all derivations of $\mathfrak{R}$, that is,

$$
\begin{gathered}
B(D X, Y)+B(X, D Y)=0, \\
T(D X, Y, Z)+T(X, D Y, Z)+T(X, Y, D Z)=0
\end{gathered}
$$

for any derivation $D$ and $X, Y, Z \in \mathbb{R}$.

An ideal $\mathfrak{M}$ in $\mathfrak{R}$ is called characteristic if $D \mathfrak{M} \subset \mathfrak{M}$ for every derivation $D$ of $\&$. Our first theorem may now be stated as follows:

Theorem 1. An element $X$ of $\&$ belongs to the radical $\Gamma$ if and only if $T(X, Y, Z)=0$ for all $Y, Z \in \mathbb{R}^{3}$

As an immediate corollary we get the following:

Received by the editors October 4, 1948 and, in revised form, November 11, 1948.

1 Ann. of Math. vol. 50 (1949) p. 68.

2 My attention has been drawn to a paper by Malcev (Bull. Acad. Sci. URSS. vol. 9 (1945) pp. 329-356) where it is shown that $\mathfrak{R}$ is an ideal.

- Since $T(X, Y, Z)=-T(Z, Y, X)$ this condition is clearly equivalent to $B(X, Y)$ $=0$ for all $Y \in \mathbb{R}^{\prime}=[R, \&]$. Professor Jacobson has kindly brought it to my notice that this theorem is contained in Cartan's thesis p. 109. 
COROLlaRY 1. Both $\Gamma$ and $\mathfrak{N}$ are characteristic ideals in $\mathfrak{R}$.

For every $N \in \mathfrak{R}$, ad $N$ is a nilpotent derivation of $\mathfrak{R}$. Hence $\sigma_{N}=\exp (\operatorname{ad} N)$ is defined and is an automorphism of $\mathbb{R}$. Let $\mathfrak{M}$ be any nilpotent ideal in $\mathfrak{R}$. Then $\mathfrak{M} \subset \mathfrak{N}$. By $G_{\mathfrak{R}}$ we denote the group of all automorphisms of $\mathfrak{R}$ of the form $\sigma_{M_{1}} \sigma_{M_{2}} \cdots \sigma_{M_{r}}$ where $M_{1}, \cdots$, $M_{r} \in \mathbb{M}$ and $r \geqq 1$. Clearly every ideal is invariant under $G_{\mathfrak{D}}$. Our second theorem now runs as follows:

THEOREM 2. Let $\mathfrak{S}$ be a semisimple subalgebra of $\mathbb{R}$ such that $\mathfrak{R}=\mathfrak{S}+\Gamma$. Then, given any semisimple subalgebra $\mathfrak{M}$ of $\mathfrak{R}$, there exists a $\sigma \in G_{\mathfrak{R}}$ such that $\mathfrak{M \subset} \subset \widetilde{\sigma}$.

The following two corollaries follow immediately from this theorem.

Corollary 2. Any maximal semisimple subalgebra of $\mathfrak{R}$ is isomorphic to $\mathbb{R} / \Gamma$.

CoROllaRy 3. Given any two maximal semisimple subalgebras $\mathfrak{S}_{1}, \mathfrak{S}_{2}$ of $\mathfrak{R}$, there exists a $\tau \in G_{\Re}$ such that $\tau \mathfrak{S}_{1}=\mathfrak{S}_{2}$.

Corollary 3 is a sharper form of a result due to Malcev."

Proof of Theorem 1. First we shall prove that for any $N \in \mathfrak{R}$, $B(N, Z)=0$ for all $Z \in R$. For any $s \geqq 1$ define $\Re_{(s)}$ by induction as follows. $\mathfrak{N}_{(1)}=\mathfrak{N}, \mathfrak{R}_{(s+1)}=\left[\mathfrak{N}, \mathfrak{N}_{(\bullet)}\right]$. Then $\mathfrak{N}_{(\diamond)}$ is an ideal in $\mathfrak{R}$ and therefore $(\operatorname{ad} N$ ad $Z) \mathfrak{R} \subset \mathfrak{N}$ and $(\operatorname{ad} N$ ad $Z) \mathfrak{N}_{(\bullet)} \subset \mathfrak{N}_{(\bullet+1)}$. Hence (ad $N$ ad $Z)^{s+1} \mathfrak{R}=\mathfrak{N}_{(s)}$. But $\mathfrak{N}$ is nilpotent and therefore $\mathfrak{R}_{(s)}=\{0\}$ for some $s$. Hence $(\operatorname{ad} N \text { ad } Z)^{s+1} Z=\{0\}$ or $(\operatorname{ad} N \text { ad } Z)^{\star+1}=0$. Therefore ad $N$ ad $Z$ is nilpotent and $s p(\operatorname{ad} N$ ad $Z)=B(N, Z)=0$.

Let $\mathfrak{M}$ be the set of all $X \in \mathbb{R}$ such that $T(X, Y, Z)=0$ for all $Y, Z \in \mathbb{R}$. Since $T$ is invariant under all derivations of $\mathbb{R}, \mathfrak{M}$ is a characteristic ideal in $\mathfrak{R}$. We have to show that $\mathfrak{M}=\Gamma$. Let $X \in \Gamma$. For any $Y \in \mathbb{R}$, ad $Y$ is a derivation of $\mathbb{R}$ and $\Gamma$ is invariant under ad $Y$. Hence ad $Y$ induces a derivation of $\Gamma$ and therefore $(\operatorname{ad} Y) \Gamma \subset \Re$. So $[X, Y]$ $=-(\operatorname{ad} Y) X \in \mathfrak{N}$. Hence $B([X, Y], Z)=T(X, Y, Z)=0$ for all $Z \in \mathbb{R}$. Since this is true for every $Y, X \in \mathbb{M}$. Hence $\Gamma \subset \mathfrak{M}$. On the other hand let $\mathfrak{M}^{\prime}=[\mathfrak{M}, \mathfrak{M}]$. Then it is clear that for any $M \in \mathfrak{M}^{\prime}, B(M, Z)=0$ for all $Z \in \mathfrak{R}$. Hence by Cartan's criterion for solvability $\mathfrak{M}^{\prime}$ is solvable. Hence $\mathfrak{M}$ is solvable and therefore $\mathfrak{M} \subset \Gamma$. So the theorem is proved.

Since $\mathfrak{M}$ is a characteristic ideal the same is true of $\Gamma$. Hence if $D$ is a derivation of $\mathbb{R}, D \Gamma \subset \Gamma$ and so $D$ induces a derivation of $\Gamma$. Therefore $D \Gamma \subset \mathfrak{N}$ and so $D \mathfrak{N} \subset D \Gamma \subset \mathfrak{N}$. Hence $\mathfrak{N}$ is also a characteristic ideal.

A. Malcev, C. R. Acad. Sci. URSS. vol. 36 (1942) p. 42. 
Proof of Theorem 2. Since $\mathfrak{S}$ is semisimple $\mathfrak{S} \cap \Gamma=\{0\}$. Hence every $P \in \mathbb{R}$ can be written uniquely as

$$
P=S(P)+\nu(P)
$$

where $S(P) \in \mathcal{S}$ and $\nu(P) \in \mathfrak{R}$. Hence

$$
\begin{aligned}
{[P, Q]=} & {[S(P), S(Q)]+[S(P), \nu(Q)] } \\
& +[\nu(P), S(Q)]+[\nu(P), \nu(Q)] .
\end{aligned}
$$

Therefore

$$
\begin{gathered}
S([P, Q])=[S(P), S(Q)], \\
\nu([P, Q])=[\nu(P), S(Q)]+[S(P), \nu(Q)]+[\nu(P), \nu(Q)] .
\end{gathered}
$$

We have already seen that $(\operatorname{ad} X) \Gamma \subset \mathfrak{R}$ for any $X \in \mathbb{R}$. Hence, $\nu([P, Q]) \in \mathfrak{R}$. Let $\nu$ denote the mapping $P \rightarrow \nu(P)$. Then $\nu([\mathfrak{M}, \mathfrak{M}])$ $\subset \mathfrak{R}$. But $\mathfrak{M}$ is semisimple. Therefore $[\mathfrak{M}, \mathfrak{R}]=\mathfrak{M}$ and $\nu(\mathfrak{R}) \subset \mathfrak{N}$. Hence $\mathfrak{M C S}+\mathfrak{R}$.

Put $\mathfrak{R}_{0}=\mathfrak{S}+\mathfrak{R}$. First suppose that $\mathfrak{R}$ is abelian. The mapping $P \rightarrow S(P)$ is a homomorphic mapping of $\mathfrak{M}$ into $\mathbb{S}$. For any $S \in \mathbb{S}$ let $D_{S}$ denote the derivation of $\mathfrak{R}$ given by $D_{S} N=[S, N](N \in \mathfrak{R})$. Then the mapping $\rho$ defined by $\rho(P)=D_{S(P)}$ is a representation of $\mathfrak{M}$. Also, since $\mathfrak{R}$ is abelian

$$
\begin{aligned}
\nu([P, Q]) & =[S(P), \nu(Q)]-[S(Q), \nu(P)] \\
& =\rho(P) \nu(Q)-\rho(Q) \nu(P) .
\end{aligned}
$$

Hence $\nu$ is a Whitehead mapping of $\mathfrak{R}$ into $\mathfrak{R}$ with respect to the representation $\rho$. Therefore by the first Whitehead lemma ${ }^{5}$ there exists an element $-N \in \mathfrak{R}$ such that $\nu(P)=-\rho(P) N=[N, S(P)]$. Hence

$$
P=S(P)+\nu(P)=S(P)+[N, S(P)]=\exp (\operatorname{ad} N) S(P)
$$

since $(\operatorname{ad} N)^{2}=0, \mathfrak{N}$ being abelian. Therefore $\mathfrak{M} \subset \sigma_{N} \subseteq$ and so the theorem is proved in this case.

Now consider the general case. Let $n=\operatorname{dim} \mathfrak{R}$. If $n \leqq 1, \mathfrak{N}$ is abelian and so the theorem is true. Hence we can assume $n>1$ and use induction on $n$. Further we can assume that $\mathfrak{R}$ is not abelian so that $\mathfrak{N}^{\prime}=[\mathfrak{N}, \mathfrak{R}] \neq\{0\}$. Let $X \rightarrow \vec{X}$ denote the natural homomorphism of $\mathfrak{R}_{0}$ onto $\overline{\mathfrak{R}}_{0}=\mathfrak{R}_{0} / \mathfrak{R}^{\prime}$. The radical of $\overline{\mathfrak{R}}_{0}$ is $\overline{\mathfrak{R}}=\mathfrak{R} / \mathfrak{R}^{\prime}$ which is abelian. Let $\overline{\mathfrak{M}}$ and $\overline{\mathfrak{S}}$ be the images of $\mathfrak{M}$ and $\mathscr{S}$ respectively in $\overline{\mathfrak{R}}_{0}$. Then they are both semisimple and

5ee Hochschild, Amer. J. Math. vol. 64 (1942) p. 677. 


$$
\overline{\mathfrak{M}} \subset \overline{\mathfrak{S}}+\overline{\mathfrak{N}} \text {. }
$$

Since $\overline{\mathfrak{R}}$ is abelian it follows from the above proof that there exists an $\bar{N} \in \overline{\mathfrak{R}}$ such that

$$
\overline{\mathfrak{M}} \subset \sigma_{\bar{N}} \overline{\mathfrak{S}} .
$$

Let $N \in \bar{N}(N \in \mathfrak{R})$. The complete inverse image of $\sigma_{\mathfrak{N}} \bar{\Im}$ in $\mathfrak{l}_{0}$ is $\mathfrak{S}_{1}+\mathfrak{R}^{\prime}$ where $\mathfrak{S}_{1}=\sigma_{N} \mathfrak{S}$. Hence

$$
\mathfrak{M} \subset \mathfrak{S}_{1}+\mathfrak{R}^{\prime}
$$

Since $\mathfrak{R}$ is nilpotent, $\operatorname{dim} \mathfrak{R}^{\prime}<\operatorname{dim} \mathfrak{R}=n$. Hence the induction hypothesis is applicable to $\mathfrak{R}_{1}=\mathfrak{S}_{1}+\mathfrak{R}^{\prime}$ and therefore there exists a $\sigma_{1} \in G_{\Re}$, such that $\mathfrak{M C} \subset \sigma_{1} \mathfrak{S}_{1}$. But $G_{\Re}, \subset G_{\Re}$ and therefore $\sigma=\sigma_{1} \sigma_{N} \in G_{\Re}$ and $\mathfrak{M C} \sigma \sigma^{\circ}$.

Now let $\mathfrak{S}^{*}$ be any maximal semisimple algebra of $\mathfrak{R}$. It follows from the above theorem that $\mathscr{S}^{*} \subset \sigma \widetilde{S}$ for some $\sigma \in G_{\Re}$. Since $\sigma$ is an automorphism of $\mathfrak{R}, \sigma \mathfrak{S}$ is semisimple. Therefore $\mathfrak{S}^{*}=\sigma \mathfrak{S} \cong \mathbb{S} \cong \mathbb{R} / \Gamma$.

If $\mathfrak{S}_{1}$ and $\mathfrak{S}_{2}$ are two maximal semisimple subalgebras of $\mathfrak{R}$, we can find $\tau_{1}, \tau_{2} \in G_{\Re}$ such that $\mathfrak{S}_{1}=\tau_{1} \mathfrak{S}, \mathfrak{S}_{2}=\tau_{2} \mathfrak{S}$. Then $\tau \mathfrak{S}_{1}=\mathfrak{S}_{2}$ where $\tau=\tau_{2} \tau_{1}^{-1} \in G_{\Re}$.

Summary. Let $\mathfrak{R}$ be a Lie algebra over a field of characteristic zero and let $\Gamma$ be its radical. It is proved that any $X \in \mathfrak{R}$ belongs to $\Gamma$ if and only if $s p(\operatorname{ad}[X, Y]$ ad $Z)=0$ for all $Y, Z \in \mathbb{R}$. Here $X \rightarrow \operatorname{ad} X$ is the adjoint representation of $\mathfrak{R}$. Further let $\mathfrak{R}$ be the maximal nilpotent ideal of $\mathfrak{R}$ and let $\mathfrak{S}$ and $\mathfrak{S}^{*}$ be any two maximal semisimple subalgebras of $\mathfrak{R}$. Then $\mathfrak{S}+\mathfrak{N}=\mathfrak{S}^{*}+\mathfrak{N}$ and $\mathfrak{S}$ and $\mathfrak{S}^{*}$ are conjugate in a certain strict sense.

INSTITUTE fOR AdVANeEd STUdy 